\title{
Characterization of FDG-PET images after stereotactic body radiation therapy for lung cancer.
}

\section{$\operatorname{AUTHOR}(S):$}

Matsuo, Yukinori; Nakamoto, Yuji; Nagata, Yasushi; Shibuya, Keiko; Takayama, Kenji; Norihisa, Yoshiki; Narabayashi, Masaru; ... Higashi, Tatsuya; Togashi, Kaori; Hiraoka, Masahiro

\section{CITATION:}

Matsuo, Yukinori ... [et al]. Characterization of FDG-PET images after stereotactic body radiation therapy for lung cancer.. Radiotherapy and oncology : journal of the European Society for Therapeutic Radiology and Oncology 2010, 97(2): 200-204

\section{ISSUE DATE:}

2010-11

URL:

http://hdl.handle.net/2433/131959

\section{RIGHT:}

(C) 2010 Elsevier Ireland Ltd; This is not the published version. Please cite only the published version.; この論文は出版社版でありません。引 用の際には出版社版をご確認ご利用ください。 
Title:

Characterization of FDG-PET Images after Stereotactic Body Radiation Therapy for Lung Cancer

\section{Authors:}

Yukinori Matsuo $^{1}$, Yuji Nakamoto ${ }^{2}$, Yasushi Nagata ${ }^{3}$, Keiko Shibuya $^{1}$, Kenji Takayama $^{1}$, Yoshiki Norihisa ${ }^{1}$, Masaru Narabayashi ${ }^{1}$, Takashi Mizowaki ${ }^{1}$, Tsuneo Saga ${ }^{4}$, Tatsuya Higashi $^{5}$, Kaori Togashi ${ }^{2}$, Masahiro Hiraoka ${ }^{1}$.

\section{Affiliations:}

1. Department of Radiation Oncology and Image-applied Therapy, Graduate School of Medicine, Kyoto University, Kyoto, Japan.

2. Department of Diagnostic Imaging and Nuclear Medicine, Graduate School of Medicine, Kyoto University, Kyoto, Japan.

3. Division of Radiation Oncology, Hiroshima University Hospital, Hiroshima, Japan.

4. Diagnostic Imaging Group, Molecular Imaging Center, National Institute of Radiological Sciences, Chiba, Japan.

5. Shiga Medical Center Research Institute, Moriyama, Japan.

\section{Corresponding author:}

Yukinori Matsuo, M.D., 
Department of Radiation Oncology and Image-applied Therapy, Graduate School of Medicine, Kyoto University, 54 Kawahara-cho, Shogoin, Sakyo-ku, Kyoto, 606-8507, Japan.

Phone: +81-75-751-3419, fax: +81-75-771-9749

E-mail: ymatsuo@kuhp.kyoto-u.ac.jp

\section{Running head:}

FDG-PET after SBRT for Lung Cancer

\section{Keywords:}

FDG-PET; Stereotactic Body Radiation Therapy; Lung cancer

This article includes 16 pages, 1 table and 3 figures. 


\begin{abstract}
:
Background and Purpose: The purpose was to characterize ${ }^{18} \mathrm{~F}$-fluorodeoxyglucosepositron emission tomography (FDG-PET) findings after stereotactic body radiation therapy (SBRT) for lung cancer.
\end{abstract}

Materials and Methods: This was a retrospective review of 32 FDG-PET scans from 23 patients who underwent SBRT for lung cancer and who showed no evidence of local recurrence. The FDG uptake by lesions was assessed visually using a 3-point scale ( 0 , none or faint; 1, mild; or 2, moderate to intense), and the demarcation (ill- or welldefined) was evaluated. For semi-quantitative analysis, the maximum standardized uptake value (SUVmax) was calculated.

Results: Grade 2 intensity was observed in 70, 33, 30, and $0 \%$ of PET scans performed $<6,6-12,12-24$, and $>24$ months, respectively, after SBRT; well-defined demarcation was observed in $80,33,40$, and $17 \%$, respectively, and the respective means of the SUVmax were 4.9, 2.6, 3.0 and 2.3. The SUVmax was significantly higher for scans performed at $<6$ months than at $6-12$ or $>24$ months.

Conclusions: FDG uptake tended to be intense and well-defined at early times after SBRT, especially within 6 months, and was faint and ill-defined at later periods. Moderate to intense FDG uptake observed soon after SBRT does not always indicate a residual tumour. 


\section{Introduction}

Stereotactic body radiation therapy (SBRT) is an important option for the treatment of solitary lung cancer, especially in inoperable patients. Initial studies on SBRT were reported by Blomgren et al. in 1995 [1] and Uematsu et al. in 1998 [2]. Thereafter, many promising outcomes have been reported for SBRT of solitary non-small cell lung cancer (NSCLC). We reported our experience in SBRT for primary lung cancer [3]. The local control rates for stage I NSCLC were $96.7 \%$ for T1a, $84.5 \%$ for T1b, and $78.1 \%$ for T2a, respectively. The excellent rates for local control of stage I NSCLC after SBRT were also reported by several authors, and they ranged from 77 to $95 \%$ [4-7].

When SBRT is applied to operable patients, the early detection of local recurrence after SBRT is vital for those who may be salvaged with surgery [8]. However, it is difficult to detect local recurrence based on computed tomography (CT) alone [9, 10], because consolidations representing radiation-induced inflammation or fibrosis [11] can overlap the tumour and prevent evaluation of local tumour status.

As a diagnostic imaging tool, positron emission tomography (PET) with ${ }^{18} \mathrm{~F}$ fluorodeoxyglucose (FDG) reveals metabolic changes. FDG-PET currently plays important roles in not only staging [12] and restaging [13] but also the prognostic assessment of lung cancer [14]. The clinical significance of FDG-PET after conventional radiotherapy for NSCLC has been described [15-18], but FDG-PET findings after SBRT are limited $[19,20]$. Even with FDG-PET, it may be difficult to differentiate between recurrence and inflammatory changes. Nevertheless, the recognition of the uptake patterns and frequency of FDG accumulation owing to inflammatory processes after SBRT would be helpful in interpreting PET images during follow-up. 
The objective of this study was to characterize the FDG-PET findings in patients with lung cancer treated by SBRT.

\section{Materials and methods}

\section{Patients}

Inclusion criteria for this study were as follows: (1) availability of at least one FDG-PET scan performed 1 or more months after SBRT and attenuation-corrected PET images reconstructed by iterative algorithms; (2) follow-up duration of >12 months; and (3) no local recurrence for at least 6 months after the FDG-PET scan. Local recurrence was diagnosed based on histological confirmation or continuous enlargement of local tumour on CT for 6 months or more. Among 96 patients who underwent SBRT for primary lung cancer at our institution between September 1998 and November 2005, 58 patients, 9 patients and 6 patients were excluded based on the criteria (1), (2) and (3), respectively. The remaining 23 patients were eligible for this study. All patients provided written informed consent for SBRT and associated researches which were approved by the institutional review board. Characteristics of the patients were shown in Table 1. Fifteen patients were male; eight were female. The median age of the patients was 77 years (range, 64-89). The histological findings were adenocarcinoma in 14 patients and squamous cell carcinoma in six patients, with unconfirmed diagnoses in three patients. The post-SBRT median follow-up duration was 51 months (range, 13-113).

\section{SBRT technique}

Details of the SBRT technique used in the present study have been described in our previous study [21]. The patient's body was immobilized using a stereotactic body 
frame (Elekta AB, Stockholm, Sweden). SBRT was planned using a commercial treatment planning system (CADPLAN or Eclipse; Varian Medical Systems, Inc., Palo Alto, CA). The planning target volume (PTV) was defined as the internal target volume, which was delineated on long scan-time CT images, with a 5-mm margin for setup uncertainty. Multiple non-coplanar static ports (5-8 ports) were arranged for the PTV. The prescription dose was $48 \mathrm{~Gy}$, administered in four fractions at the isocenter. Irradiation was performed with 6-MV X-ray beams from a linear accelerator (Clinac 2300 CD; Varian Medical Systems). The median overall treatment time was 11 days (range, 4-14) in this cohort.

\section{PET scanning}

${ }^{18}$ F-FDG was synthesized by the nucleophilic substitution method, using an ${ }^{18} \mathrm{~F}$ FDG synthesizing instrument (F-100; Sumitomo Heavy Industries, Tokyo, Japan) and a cyclotron (CYPRIS-325R; Sumitomo Heavy Industries, Tokyo, Japan). Patients fasted for $\geq 4 \mathrm{~h}$ before the intravenous injection of approximately $370 \mathrm{MBq}$ of FDG. Wholebody PET images with attenuation correction were acquired about 50 min later, using a whole-body PET scanner with an 18-ring detector arrangement (Advance; GE Healthcare, Milwaukee, WI). The system permitted the simultaneous acquisition of 35 transaxial images with a $4.25-\mathrm{mm}$ interslice spacing. The transaxial resolution was 4.2 $\mathrm{mm}$ at full width at half maximum, allowing for multidirectional reconstruction of the images without loss of resolution. The field of view and pixel size of the reconstructed images were $128 \mathrm{~mm}$ and $4 \mathrm{~mm}$, respectively. The images were reconstructed by orderedsubsets expectation maximization algorithm. 


\section{Evaluation and analysis}

There were a total of 32 FDG-PET scans for the 23 patients, because nine patients each had two PET scans. For the analysis, the PET scans were divided into four groups according to the time duration between the completion of SBRT and the PET scan, which ranged from 1 to 51 months with a median of 12 months: $<6$ months, 10 scans; 6-12 months, six scans; $12-24$ months, 10 scans; and >24 months, six scans. The intensity and pattern of the FDG uptake were evaluated qualitatively and semiquantitatively in the pulmonary region, which received a high dose.

For the qualitative evaluation, the intensity of the FDG uptake was assessed visually using a 3-point scale: 0 , none or faint uptake; 1 , mild uptake, comparable to that in the blood pool; and 2, moderate to intense uptake, greater than that in the blood pool. The demarcation of the tracer uptake was categorized as well- or ill-defined. The qualitative evaluations were determined by a board-certified radiologist and nuclear medicine physician (YN) and a board-certified radiation oncologist (YM) on consensus without any information about time duration between SBRT and FDG-PET. For the semiquantitative analysis, we calculated the maximum standardized uptake value (SUVmax) after setting regions of interest. For three scans, although qualitative analysis was possible, a reliable SUVmax could not be obtained because of artifacts. Thus, a total of 29 PET scans were analyzed semi-quantitatively. The SUVmax was calculated as follows:

$\mathrm{SUV}=\mathrm{FDG}_{\text {region }} /\left(\mathrm{FDG}_{\text {dose }} / \mathrm{WT}\right)$, where $\mathrm{FDG}_{\text {region }}$ is the decay-corrected regional ${ }^{18} \mathrm{~F}$ FDG concentration in $\mathrm{Bq} / \mathrm{ml}, \mathrm{FDG}_{\mathrm{dose}}$ is the injected ${ }^{18} \mathrm{~F}-\mathrm{FDG}$ in $\mathrm{Bq}$, and WT is the body weight in grams.

Statistical significance was defined as $p<0.05$. 


\section{Results}

\section{Qualitative evaluation (Fig. 1)}

The numbers of PET scans showing FDG uptake intensity grades of 0,1 , and 2 for each time category were: $1(10 \%), 2(20 \%)$, and $7(70 \%)$ for scans performed at $<6$ months; $2(33 \%), 2(33 \%)$, and $2(33 \%)$ at 6-12 months; $2(20 \%), 5(50 \%)$, and $3(30 \%)$ at 12-24 months; and $1(17 \%), 5(83 \%)$, and 0 at $>24$ months. Uptakes with ill-defined and well-defined demarcations were observed in $2(20 \%)$ and $8(80 \%)$ scans at $<6$ months; $4(67 \%)$ and $2(33 \%)$ scans at 6-12 months, $6(60 \%)$ and $4(40 \%)$ scans at $12-24$ months, and $5(83 \%)$ and $1(17 \%)$ scans at $>24$ months. The FDG uptake tended to be intense and well-defined at early times after SBRT.

\section{Semi-quantitative evaluation (Fig. 2)}

A moderate negative correlation was observed between the SUVmax and time after SBRT (Pearson's product-moment correlation coefficient $=-0.388 ; p=0.038$ ). The SUVmax (mean $\pm \mathrm{SD}$ ) was $4.9 \pm 2.2$ at $<6$ months, $2.6 \pm 1.5$ at $6-12$ months, $3.0 \pm 0.9$ at 12-24 months, and $2.3 \pm 0.9$ at $>24$ months. The SUVmax at $<6$ months was significantly higher than that at 6-12 months or $>24$ months ( $p=0.042$ or 0.020 , Tukey HSD test). Differences in the SUV max were not significant between 6-12 months, 12-24 months and $>24$ months.

\section{Changes in FDG uptakes between two scans}

Table 2 shows FDG-PET findings in the nine patients who had two PET scans. FDG uptakes in three of the nine patients (Patients A, D and F in Table 2) changed into typical findings (i.e. faint intensity, ill-defined demarcation, and less SUVmax) in the later periods. A representative case is demonstrated in Fig. 3. No significant change was observed in two patients (Patients B and C). The remaining 4 patients (Patients E, G, H 
and I) showed a higher grade of intensity or a higher SUVmax in the second scan than in the first scan.

\section{Discussion}

Two previous reports have described FDG-PET findings after SBRT for lung cancer. Ishimori et al. investigated the feasibility of PET with ${ }^{18} \mathrm{~F}-\mathrm{FDG}$ and ${ }^{11} \mathrm{C}$ methionine (MET) in nine patients treated with SBRT [19]. The SUV decreased gradually with time in five patients, whereas it increased at 2 weeks after SBRT in two patients and at $>3$ months after SBRT in the remaining two patients. Radiation pneumonitis was thought to be the cause of this increase; the addition of MET-PET did not supply any information beyond that provided by FDG-PET. Hoopes et al. also evaluated FDG-PET in patients treated with SBRT [20]. PET analysis was performed in 28 patients after a median post-SBRT time of 17.3 months (range, 4-48). Four of the 28 patients showed a high SUV (2.5-5.87) without evidence of local, nodal, or distant failure. In the present study, the SUVmax remained high (mean, 2.7; range, 1.3-4.3) at $\geq 12$ months after SBRT, which is consistent with the results of Hoopes et al. [20].

It is important to detect local recurrence soon after SBRT, but this is difficult based on CT alone. We have previously evaluated post-irradiation changes and local recurrence after SBRT, based on CT [9]. However, in the previous study, we could not detect any significant CT differences between radiation-induced inflammatory changes and local recurrence. We concluded that early detection of local recurrence is difficult using CT because of the dense consolidation, called mass-like consolidation, which was observed at a median post-SBRT time of 5 months in $68 \%$ of the cases. Most cases (89\%) of consolidation were confirmed as radiation-induced lung injury, although a few (11\%) were local recurrence. Takeda et al. reported that 20 of 50 patients had abnormal opacity 
that was suspicious for local recurrence at a median of 20.7 months after SBRT [10]. However, only three patients had recurrence, and the remaining 17 patients were free from recurrence or were considered equivocal. They also concluded that it was difficult to distinguish radiation fibrosis from local tumour recurrence.

The value of FDG-PET in detecting residual or recurrent NSCLC after conventional radiotherapy has been evaluated by Frank et al. [15] and Bury et al. [16], who found a sensitivity and specificity of $100 \%$ and $89-92 \%$, respectively. Inoue et al. have suggested a threshold SUV of 5.0 for the differential diagnosis between local recurrence of lung cancer and post-treatment changes [17]. Indeed, our study had no case in which the SUVmax was $>5.0$ at $\geq 6$ months after SBRT. According to Takeda et al. [10], a patient with a SUVmax of 5.0 at 12 months after SBRT developed local recurrence, and three patients with a SUVmax of 2.2-3.13 showed no evidence of local recurrence. However, Hoopes et al. [20] reported that two patients with a SUV >5.0 at 23-26 months were free from local recurrence. Further studies are needed to investigate an optimal SUV for distinguishing local recurrence from post-SBRT changes. As far as we are aware, there have been no studies to date regarding FDG-PET detection of local recurrence after SBRT.

The present study has some limitations. First, the study was not prospective but was a retrospective review. FDG-PET was performed in a limited number of patients, some of whom had a suspicious consolidation upon CT, which might have caused selection bias. Second, local control was not based on pathological confirmation but on CT images. Therefore, FDG uptake demonstrating recurrent tumours might have been included with that owing to inflammation caused by irradiation. However, clinically suspicious recurrent cases were not included, and a median follow-up duration of 51 months was sufficient to establish no recurrence of local tumours. 
In conclusion, this study showed that FDG uptake tended to be high and welldefined during an early time period after SBRT, especially within the initial 6 months, and became lower and ill-defined during later periods. Moderate to intense FDG uptake observed during an early period after SBRT does not always indicate residual or recurrent tumour. These findings may help in interpreting FDG-PET data for follow-up in patients with NSCLC after SBRT.

\section{Acknowledgements:}

This work was supported by Grants-in-Aid for Scientific Research No. 20229009 and 21791188 of the Ministry of Education, Culture, Sports, Science and Technology, and by Grants-in-Aid No. H20-S5 and H20-020 of the Ministry of Health, Labour and Welfare in Japan.

\section{Conflict of Interest Notification}

Yukinori Matsuo: None

Yuji Nakamoto: None

Yasushi Nagata: None

Keiko Shibuya: None

Kenji Takayama: None

Yoshiki Norihisa: None

Masaru Narabayashi: None

Takashi Mizowaki: None

Tsuneo Saga: None

Tatsuya Higashi: None

Kaori Togashi: None 
Masahiro Hiraoka: None 


\section{References:}

1. Blomgren H, Lax I, Näslund I, Svanström R. Stereotactic high dose fraction radiation therapy of extracranial tumors using an accelerator. Clinical experience of the first thirty-one patients. Acta Oncol 1995;34: 861-870.

2. Uematsu M, Shioda A, Tahara K et al. Focal, high dose, and fractionated modified stereotactic radiation therapy for lung carcinoma patients: a preliminary experience. Cancer 1998;82: 1062-1070.

3. Matsuo Y, Shibuya K, Nagata Y et al. Prognostic factors in stereotactic body radiation therapy for non-small-cell lung cancer. Int J Radiat Oncol Biol Phys in press 4. Timmerman R, McGarry R, Yiannoutsos C et al. Excessive toxicity when treating central tumors in a phase II study of stereotactic body radiation therapy for medically inoperable early-stage lung cancer. J Clin Oncol 2006;24: 4833-4839

5. Hoyer M, Roed H, Hansen AT et al. Prospective study on stereotactic radiotherapy of limited-stage non-small-cell lung cancer. Int J Radiat Oncol Biol Phys 2006;66: S128-S135

6. Zimmermann FB, Geinitz H, Schill S et al. Stereotactic hypofractionated radiotherapy in stage I (T1-2 N0 M0) non-small-cell lung cancer (NSCLC). Acta Oncol 2006;45: 796-801

7. Koto M, Takai Y, Ogawa Y et al. A phase II study on stereotactic body radiotherapy for stage I non-small cell lung cancer. Radiother Oncol 2007;85: 429-434 8. Bradley J. Radiographic response and clinical toxicity following SBRT for stage I lung cancer. J Thorac Oncol 2007;2: S118-S124

9. Matsuo Y, Nagata Y, Mizowaki T et al. Evaluation of mass-like consolidation after stereotactic body radiation therapy for lung tumors. Int J Clin Oncol 2007;12: 356362 
10. Takeda A, Kunieda E, Takeda T et al. Possible misinterpretation of demarcated solid patterns of radiation fibrosis on CT scans as tumor recurrence in patients receiving hypofractionated stereotactic radiotherapy for lung cancer. Int J Radiat Oncol Biol Phys 2008;70: 1057-1065

11. Libshitz HI, Shuman LS. Radiation-induced pulmonary change: CT findings. J Comput Assist Tomogr 1984;8: 15-19

12. Silvestri GA, Gould MK, Margolis ML et al. Noninvasive staging of non-small cell lung cancer: ACCP evidenced-based clinical practice guidelines (2nd edition). Chest 2007;132: 178S-201S

13. Juweid ME, Cheson BD. Positron-emission tomography and assessment of cancer therapy. N Engl J Med 2006;354: 496-507

14. Berghmans T, Dusart M, Paesmans M et al. Primary tumor standardized uptake value (SUVmax) measured on fluorodeoxyglucose positron emission tomography (FDGPET) is of prognostic value for survival in non-small cell lung cancer (NSCLC): a systematic review and meta-analysis (MA) by the European Lung Cancer Working Party for the IASLC Lung Cancer Staging Project. J Thorac Oncol 2008;3: 6-12

15. Frank A, Lefkowitz D, Jaeger S et al. Decision logic for retreatment of asymptomatic lung cancer recurrence based on positron emission tomography findings. Int J Radiat Oncol Biol Phys 1995;32: 1495-1512

16. Bury T, Corhay JL, Duysinx B et al. Value of FDG-PET in detecting residual or recurrent nonsmall cell lung cancer. Eur Respir J 1999;14: 1376-1380

17. Inoue T, Kim EE, Komaki R et al. Detecting recurrent or residual lung cancer with FDG-PET. J Nucl Med 1995;36: 788-793

18. van Loon J, Grutters J, Wanders R et al. Follow-up with (18)FDG-PET-CT after radical radiotherapy with or without chemotherapy allows the detection of potentially 
curable progressive disease in non-small cell lung cancer patients: A prospective study.

Eur J Cancer 2008;45: 588-595

19. Ishimori T, Saga T, Nagata Y et al. 18F-FDG and 11C-methionine PET for evaluation of treatment response of lung cancer after stereotactic radiotherapy. Ann Nucl Med 2004;18: 669-674

20. Hoopes DJ, Tann M, Fletcher JW et al. FDG-PET and stereotactic body radiotherapy (SBRT) for stage I non-small-cell lung cancer. Lung Cancer 2007;56: 229234

21. Takayama K, Nagata, Y, Negoro, Y et al. Treatment planning of stereotactic radiotherapy for solitary lung tumor. Int J Radiat Oncol Biol Phys 2005;61: 1565-1571 


\section{Figure legends:}

Fig. 1 Visual analysis of FDG uptake regarding intensity (a) and demarcation (b).

Intensity was classified into three grades: 0 , none or faint uptake; 1, mild uptake, comparable to that in the blood pool; and 2, moderate to intense uptake, greater than that in the blood pool. The prevalence of intense and well-defined uptake gradually decreased with time after treatment.

Fig. 2 SUVmax in FDG-PET and the time duration between SBRT and FDG-PET.

(a) Scatter plot of SUVmax versus time after SBRT, showing a moderately negative correlation coefficient of -0.388 .

(b) Mean (diamond) and SD (vertical bar) of SUVmax for FDG-PET performed at different times after SBRT: <6 months, 6-12 months, 12-24 months, and >24 months. The SUVmax at $<6$ months was significantly higher than that at 6-12 or $>24$ months.

Fig. 3. 79-year-old woman (Patient D in Table 2) with cT2NOM0 primary lung adenocarcinoma, who underwent SBRT with a prescription dose of 48 Gy administered in four fractions at the isocenter

(a) CT image before treatment (left) and dose distribution of SBRT (right). The inner thin dashed, solid, and outer dashed lines indicate 48, 40, and 20-Gy isodose lines, respectively.

(b) CT and FDG-PET images 2 months after SBRT. Radiation pneumonitis is observed upon CT (arrowhead). The FDG-PET scan shows well-defined and intense (grade 2) uptake (arrows) with the SUV max of 7.1. 
(c) CT at 52 months and FDG-PET at 49 months; CT shows a scar-like shadow

(arrowhead). Ill-defined and mild (grade 1) uptake is seen upon FDG-PET (arrows). The SUVmax was 3.0. 


\section{Tables:}

Table 1. Patient characteristics

Sex

Male

Female

8

Age (median; range)

77; 64-89

Histology

Adenocarcinoma

Squamous cell carcinoma 
Table 2. FDG-PET findings in patients who received PET scan twice.

\begin{tabular}{ccccc}
\hline \multirow{2}{*}{ Patient } & $\begin{array}{c}\text { Months } \\
\text { after SBRT }\end{array}$ & $\begin{array}{c}\text { Intensity } \\
\text { grade }\end{array}$ & Demarcation & SUVmax \\
\hline \multirow{2}{*}{ A } & 37 & 1 & well & 1.3 \\
& 51 & 1 & ill & 1.3 \\
\hline \multirow{2}{*}{ B } & 21 & 1 & ill & NA \\
& 46 & 1 & ill & 3.6 \\
\hline \multirow{2}{*}{ C } & 2 & 0 & ill & 1.5 \\
& 9 & 0 & ill & 1.6 \\
\hline \multirow{2}{*}{ D } & 2 & 2 & well & 7.1 \\
& 49 & 1 & ill & 3.0 \\
\hline \multirow{2}{*}{ E } & 13 & 1 & ill & 2.6 \\
& 24 & 1 & ill & 3.1 \\
\hline \multirow{2}{*}{ F } & 1 & 2 & well & 5.1 \\
& 14 & 1 & well & 2.5 \\
\hline \multirow{2}{*}{ G } & 1 & 1 & well & 2.1 \\
& 13 & 2 & well & 3.8 \\
\hline \multirow{2}{*}{ H } & 2 & 1 & ill & 2.7 \\
& 12 & 2 & ill & 4.7 \\
\hline \multirow{2}{*}{ I } & 11 & 1 & ill & 3.1 \\
& 23 & 2 & ill & 4.3 \\
\hline
\end{tabular}

Abbreviations: $\mathrm{SBRT}=$ stereotactic body radiation therapy, $\mathrm{SUVmax}=$ maximum standardized uptake value, $\mathrm{NA}=$ not available

Intensity grades were the same as in Figure 1. 


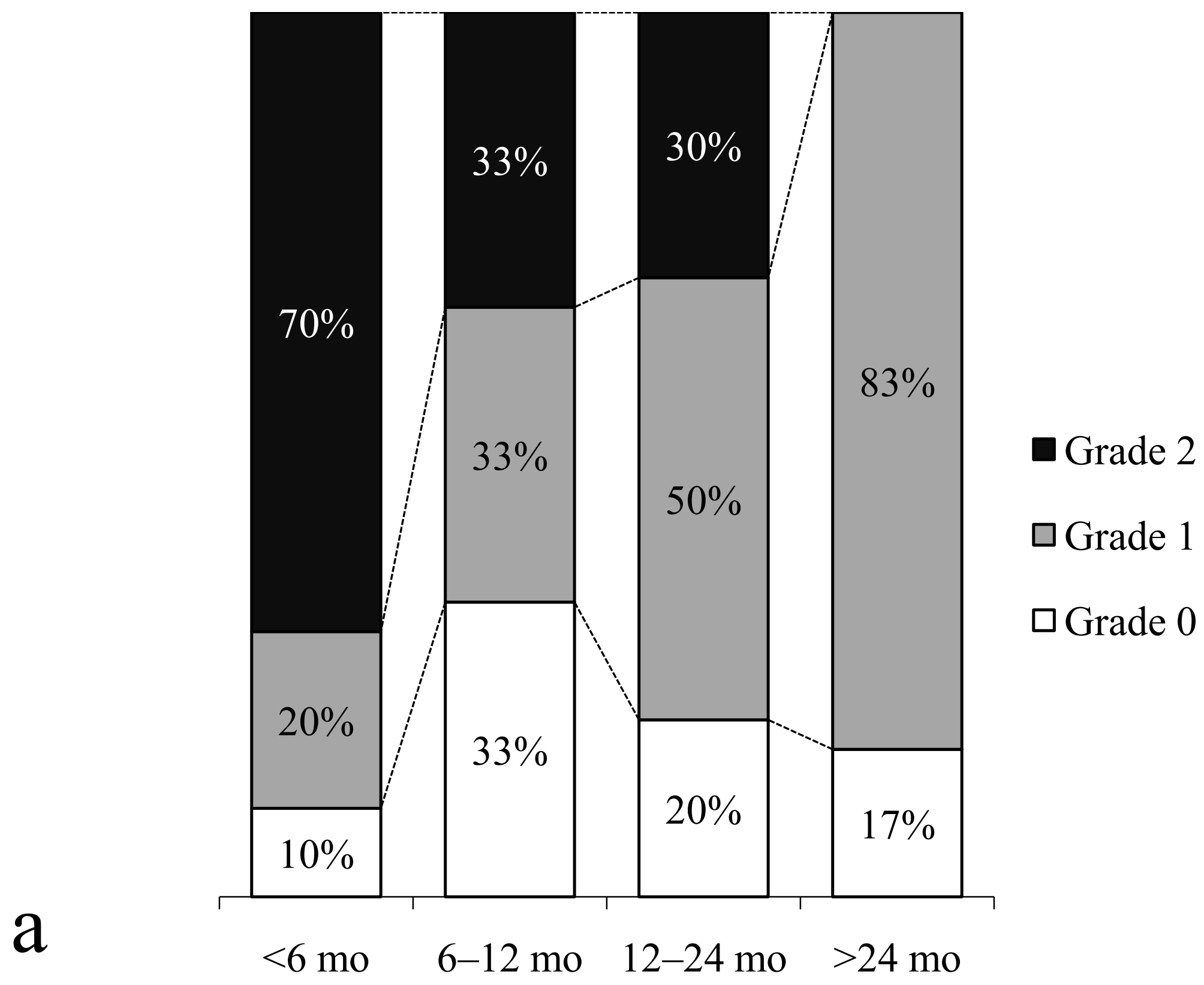




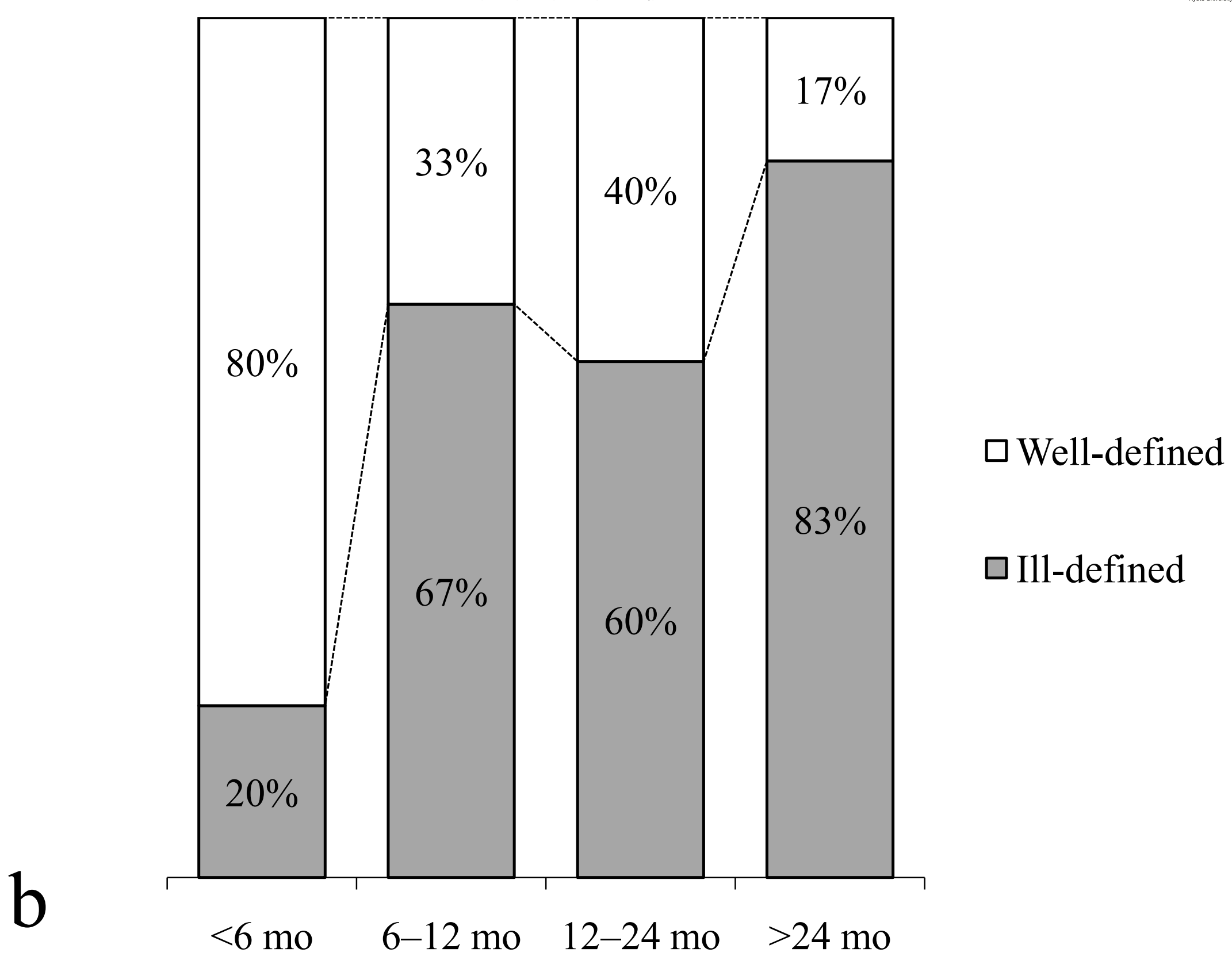




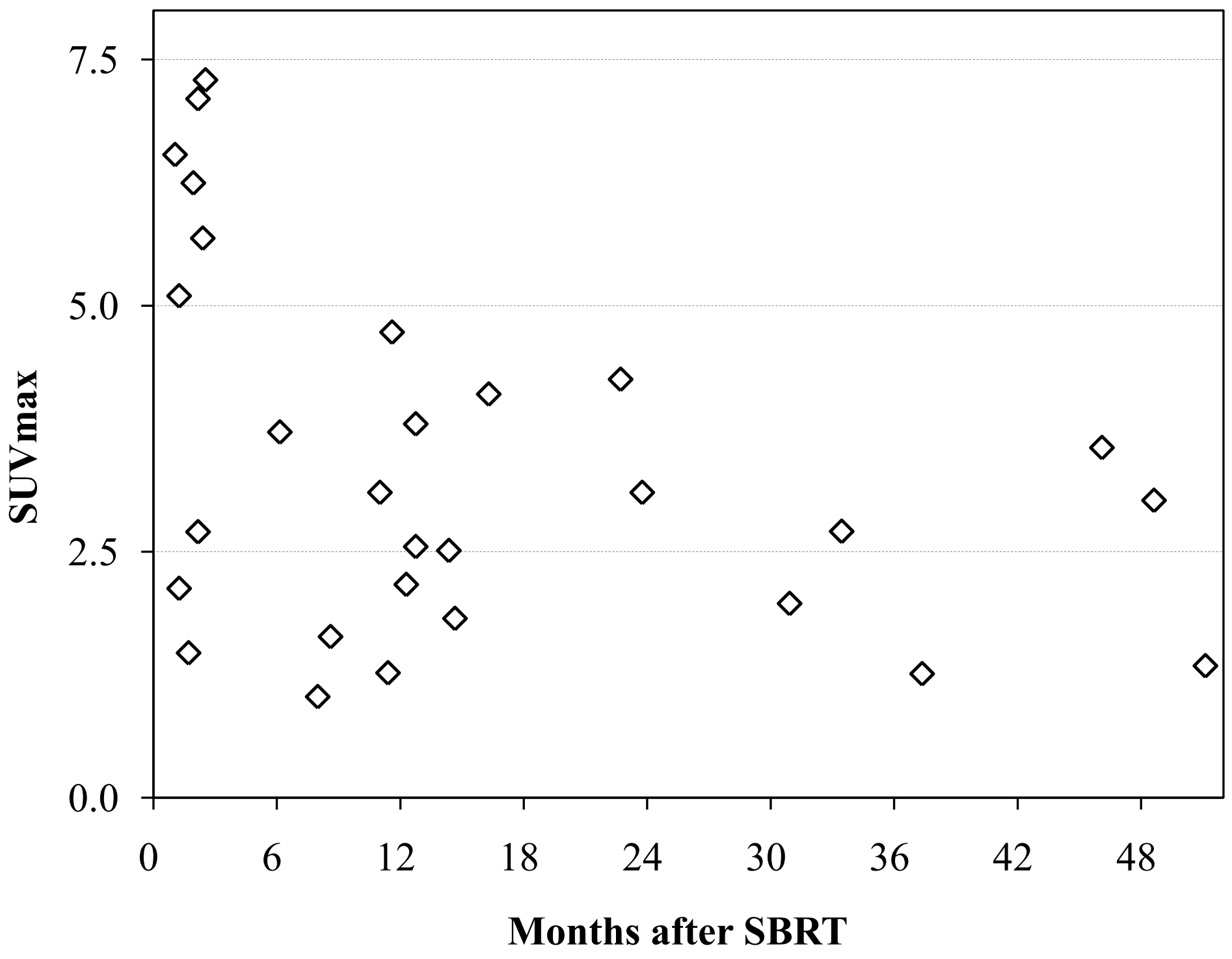

a 


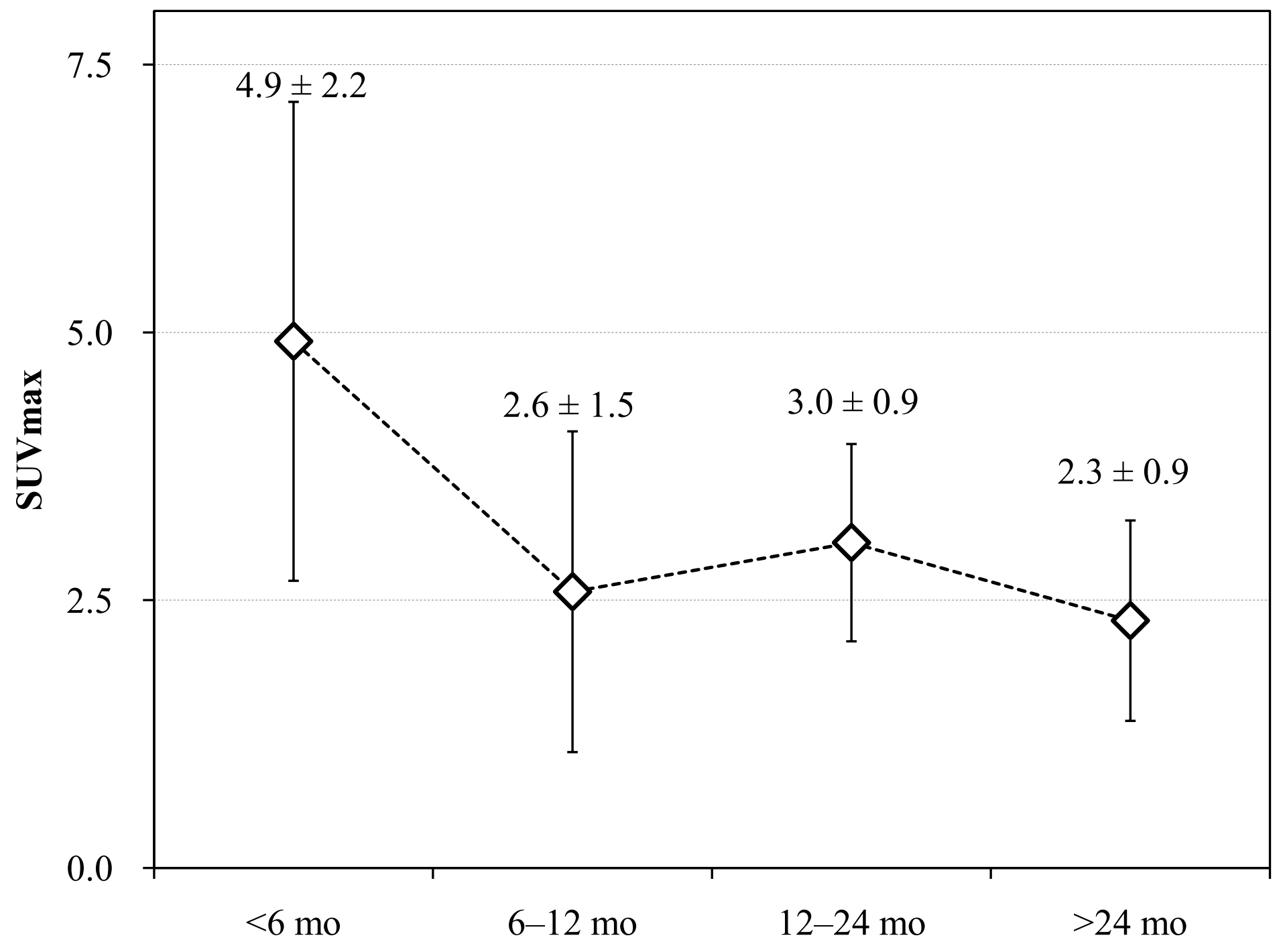

b

\section{Months after SBRT}




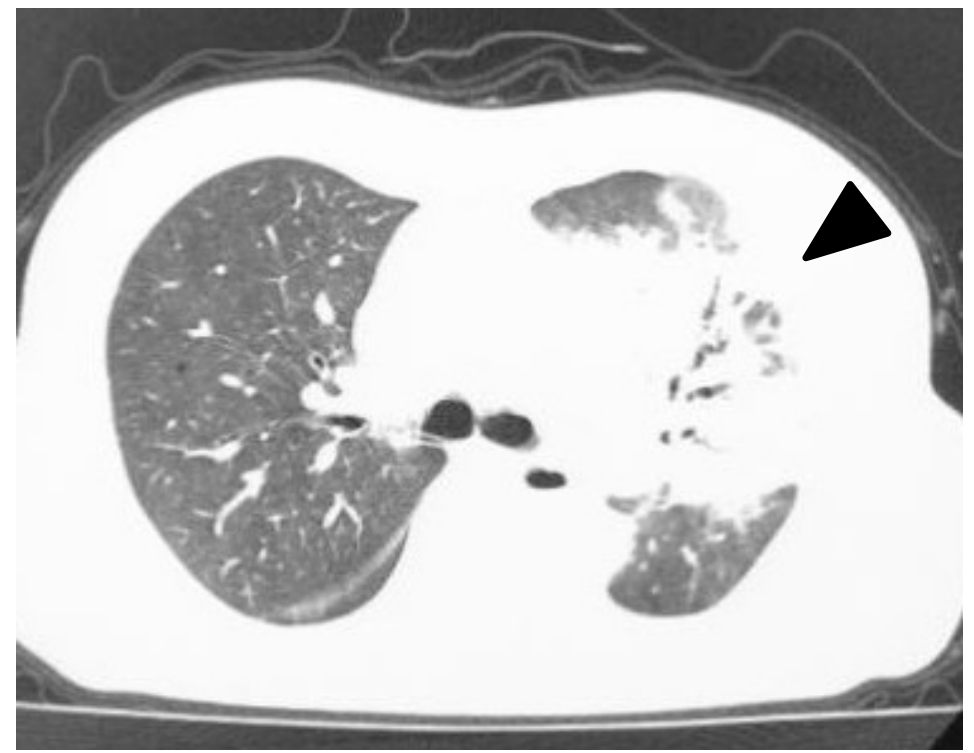

CT (axial)
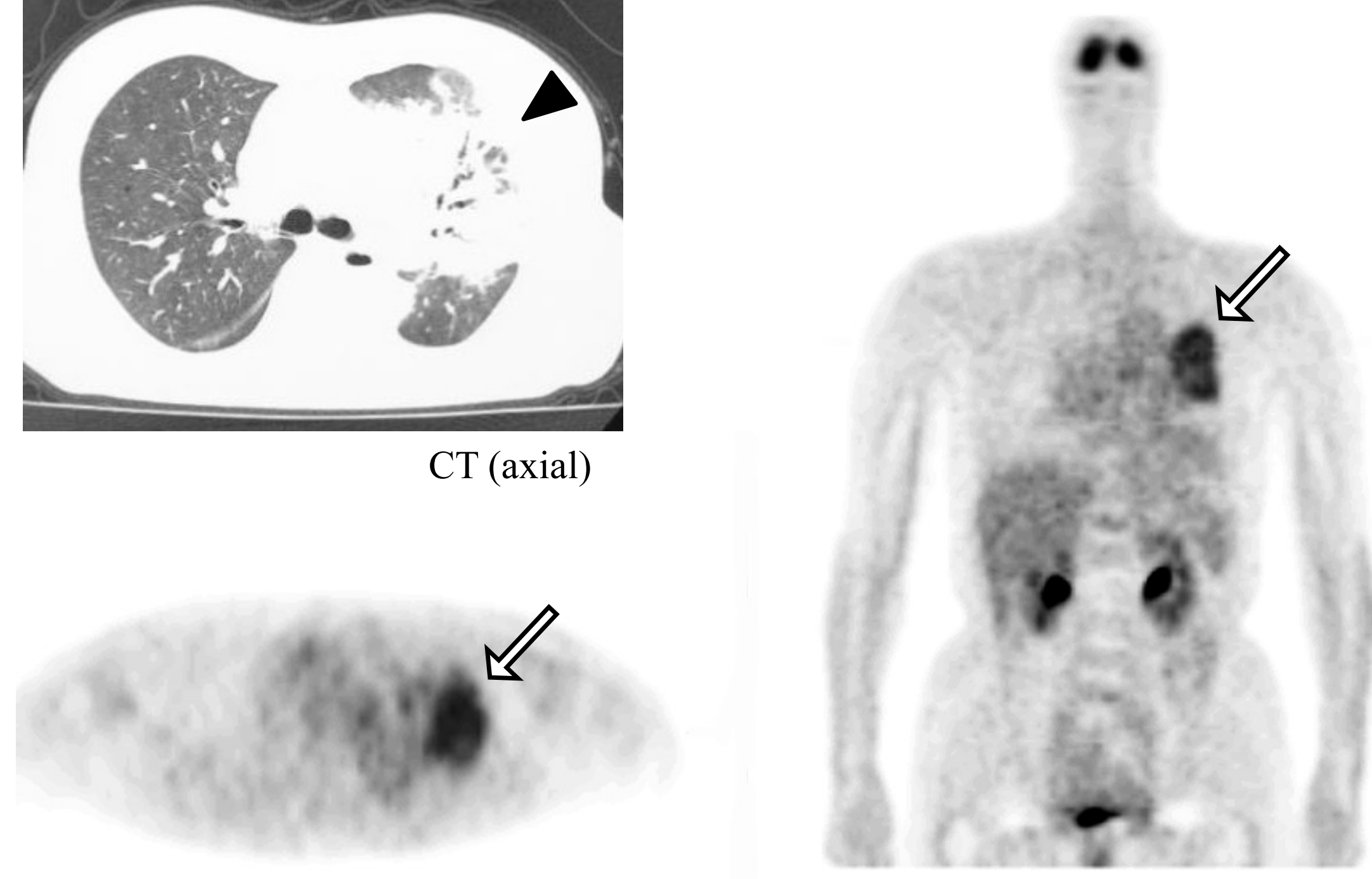

b

PET (axial)

PET (coronal) 

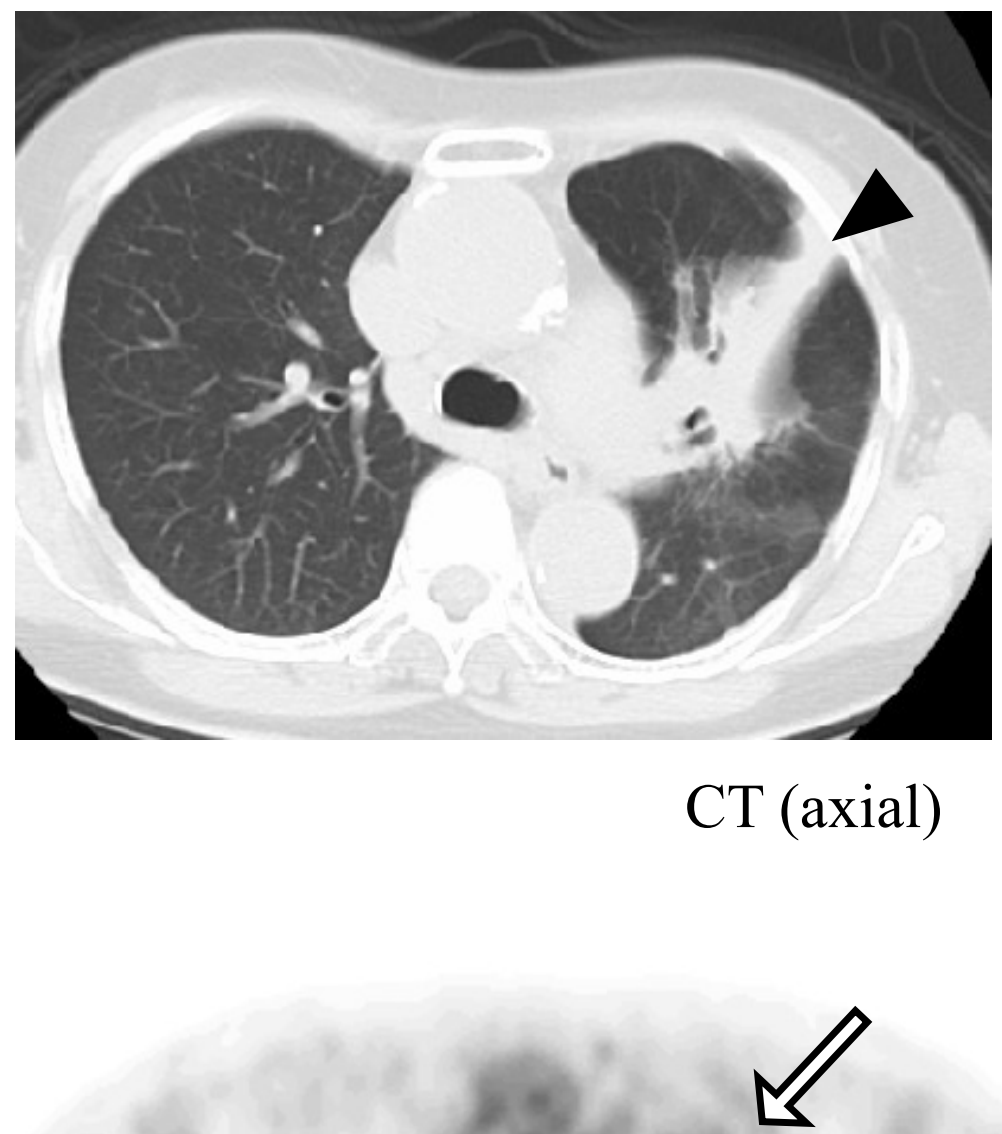

C

PET (axial)

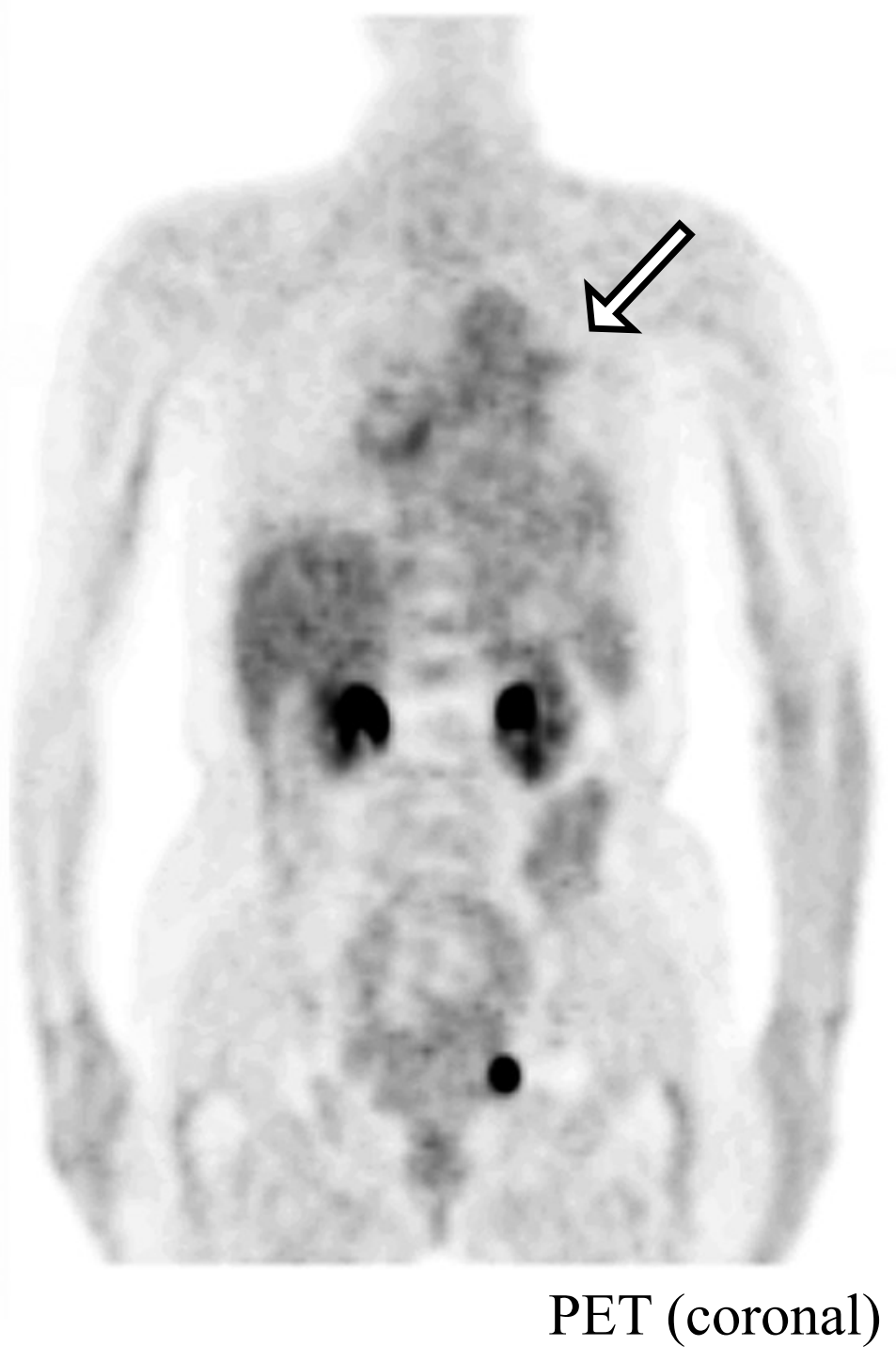

